\title{
Fast Readout and Low Power Consumption in Capacitive Touch Screen Panel by Down-sampling
}

\author{
Shuo Gao, Student Member, IEEE, Jackson Lai, and Arokia Nathan, Fellow, IEEE
}

\begin{abstract}
This paper reports on down-sampling based techniques to achieve low power consumption and fast readout for capacitive touch screen panels (TSPs). Here, touch interactivity is processed as an image, which is down-sampled and reconstructed to estimate the touch position. After the reconstruction, a regional scan is performed around the reconstructed touch location to retrieve accurate touch information. Based on experimental and simulation results, we successfully decreased readout time and power consumption by $11.3 \mathrm{~ms}$ $(68 \%)$ and $8.79 \mathrm{~mW}(68.7 \%)$, respectively, when only $25 \%$ sensors were selected. The presented technique yields higher responsivity and lower power consumption while maintaining detection accuracy.
\end{abstract}

Index Terms-down-sampling, capacitive touch screen panel, fast readout, low power consumption.

\section{INTRODUCTION}

$\mathrm{C}$ APACITIVE based touch sensing has become an ubiquitous technology in displays [1]. To design a capacitive touch screen panel (TSP), many factors need to be taken into consideration, such as power consumption, readout speed and detection accuracy. Current commercial TSPs in personal digital assistants (PDAs) can provide good accuracy and responsivity. However, short battery lifetime caused by high power consumption brings inconvenience to users, leading to the popularity of mobile power pack in market. Here, power consumption is attributed to noise and number of scanned touch sensors (in this paper, "sensor" refers to an electrode pad in multi-pad architecture and electrode intersections in rows-and-columns architecture). The noise can lead to mis-registration (in terms of presence and position of the touch signal), thus high excitation voltage is normally used, resulting in high power consumption. The latter has a positive linear correlation with power consumption. Hence, the power consumption can be reduced by removing noise and/or down-sampling the touch sensors (e.g. from $80 \times 80$ to $40 \times 40$ ). However, it should be noted that, directly reducing the number of scanned sensors adversely affects detection accuracy.

In our previous work, touch event related frames are processed as images [2]-[21], and the noise is eliminated by correlated double sampling (CDS) and spatial low-pass filtering related techniques [22][23]. In this paper, by employing the sparse and low spatial frequency property of touch signal, compressive sensing [24]-[41] and averaging based down-sampling techniques are presented to reduce power consumption while maintaining detection accuracy. Furthermore, as the number of scanned touch sensors is reduced, the touch panel's readout speed is also boosted. As illustrated in Fig. 1, instead of scanning all the sensors, only a portion of them are selected with a random or fixed pattern. A compressive sensing or low-pass spatial filtering reconstruction algorithm is then applied to the down-sampled signal, depending on the down-sampling pattern. However, after interpretation of the signal, the detected touch position may differ from the original one, thus requiring a suitable regional scan around the reconstructed touch position to retrieve accurate touch information. The regional scan range is determined by the reconstruction quality, which mainly depends on the percentage of the sampled sensors and the touch signal property. Through this method fewer sensors are scanned, therefore the readout speed is boosted and power consumption lowered.

This paper is structured as follows: in Section II, the parameters of the test bed are provided and the down-sampling based algorithms are explained in detail. Experimental and simulation results with corresponding discussions can be found in Section III.

\section{II.EXPERIMENTAL TEST BED AND ALGORITHM DESCRIPTION}

\section{Test Bed Description}

The experiments were carried out on an $80 \times 80$ touch screen panel. Details of the test bed are provided in Table I.

TABLE I

PARAMETERS OF TEST BED

\begin{tabular}{ccc}
\hline \hline Parameter & Unit & Value \\
\hline Diagonal & Inch & 10.1 \\
Aspect Ratio & None & $16 / 9$ \\
Excitation Voltage & Volt & 10 \\
Display Pixel Size & (Micrometer $^{2}$ & $56 \times 56$ \\
TX Electrode Size & Millimeter & 3 \\
RX Electrode Size & Micrometer & 449 \\
Refresh rate & Hertz & 60 \\
Sensing Array Size & (Millimeter) $^{2}$ & $3 \times 3$ \\
Sensing Array Spacing & Millimeter & 2 \\
\hline *Note: TX and RX represent transmitter and receiver respectively.
\end{tabular}




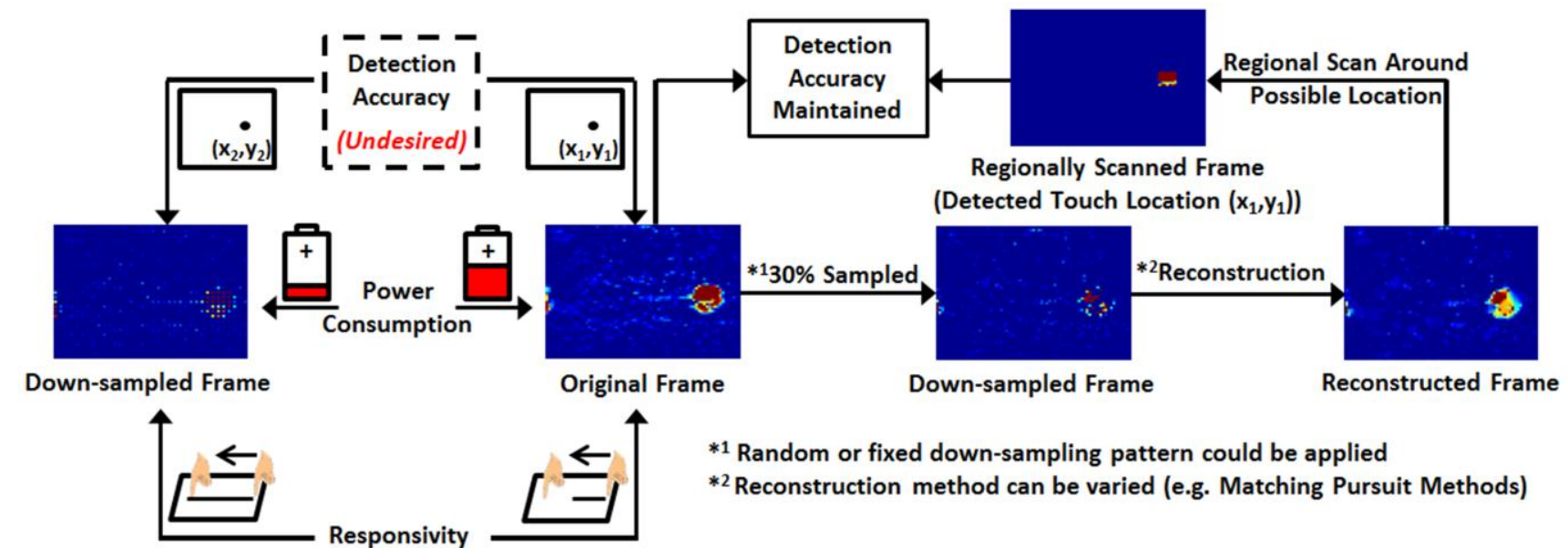

Fig. 1. Merits and drawbacks of the down-sampled signal, and compressive sensing based fast readout technique.

\section{Algorithm Description}

Instead of scanning all electrode pads or intersections, only a small portion (e.g. 25\%) were selected and measured, and then the collected data was processed by the reconstruction algorithms to recover the touch event related image. However, the touch location of the reconstructed signal may differ from the original one. The shifted distance mainly depends on both the percentage of the electrode pads sensed by the processor and the reconstruction algorithm. A pixel is defined as the unit of distance as shown in Fig. 2 (a). Thus, considering the changed distance, a regional scan within a certain range is performed to acquire the accurate touch location and relevant information. For example, if the changed distance is $d$, then the regional scan range will be a square centered at the reconstructed touch position, with a side length of $2 d$. This is illustrated in Fig. 2 (b). In this case, the side length is 2, and the square covers 9 electrode pads, which indicates that these 9 electrode pads will be scanned after the reconstruction to obtain accurate touch information.

The flowchart of the algorithm is described in Fig. 3. After selecting a portion of sensors, the scanned frame $\left(f_{\text {scan }}\right)$ is applied with reconstruction (e.g. minimum $\ell_{1}$ norm) method,

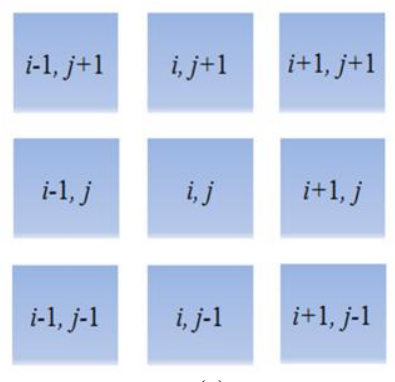

(a)

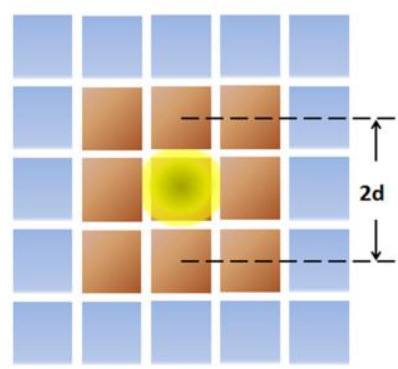

(b)
Fig. 2. (a) The distance from one pixel $(i, j)$ to other pixels $($ e.g. $(i+1, j+1))$ can be expressed by Euclidean distance. The unit of the distance is pixel. (b) Regional scan method for the multi-pad capacitance TSP. Red pads will be scanned rapidly after the reconstruction. The yellow point is the reconstructed touch position.

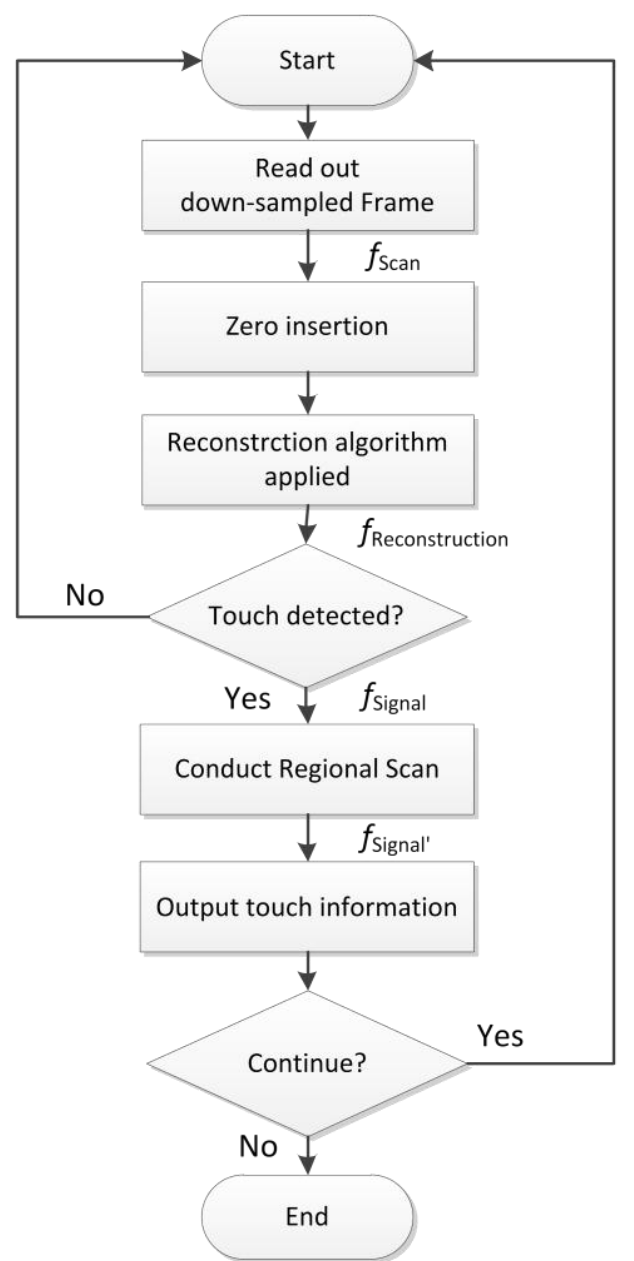

Fig. 3. Flowchart of the down-sampling based fast readout technique.

yielding the frame $f_{\text {reconstruction }}$. The reconstructed frame is then sent into the touch decision function to determine if a touch happens or not. On a positive determination, a regional scan is performed to obtain the accurate touch location and relevant touch information. 


\section{RESULTS AND DISCUSSION}

\section{A. Experimental and Simulation Results by Random Pattern Down-sampling}

Three sets of simulations were investigated by randomly collecting $10 \%, 30 \%$, and $50 \%$ of the original electrode pads. The Monte Carlo method was applied to have the distribution of the estimated touch positions. Each set had 1000 simulations. Three reconstruction results are depicted in Fig. 4 as examples. As expected, the least sampled data offered the highest probability of poor reconstruction result. The reconstructed touch positions followed a Gaussian distribution, and the distribution of the $10 \%$ sampled set is shown in Fig. 5 (a). Taking the cross-section of the direction with the largest variance (Fig. 5 (b)), we analyze the probability of the changed distance modeled as:

$$
f(d)=101.4 \exp \left(-((d) / 2)^{2}\right)
$$

Here $d$ is the distance between the reconstructed touch position and the original touch position. We assume that the reconstructed results out of $[\mu-3 \sigma, \mu+3 \sigma]$ ( $\mu$ and $\sigma$ are the mean and variance of the Gaussian distribution) [42] rarely happens, thus the maximum changed distance is 5 pixels. Under the same analysis, the maximum changed distances of $30 \%$ and $50 \%$ sampled data sets in our simulations were 4 and 3 pixels, respectively. To ensure the regional scan range can cover the original touch location, the scan side length is determined to be twice the maximum changed distance. The number of actual covered sensors within the square is expressed as:

$N_{\text {Regional-scan }}=(2 d+1)^{2}$;

where $N_{\text {Regional-scan }}$ is the number of measured sensors at the regional scan stage. Increasing the percentage of the sensed sensors gives rise to a higher probability of maintaining the touch location.

For example, Taking $10 \%$ sampled data to acquire the accurate single touch position, only 121 electrode pads centered at the peak touch value location are required to be regionally scanned. The number of totally measured sensors is 761 . In contrast, 6400 sensors are needed to be read using the traditional method. When multi-touch occurs, the number of measured sensors is expressed as:

$$
\begin{aligned}
& N_{\text {Total }}=N_{\text {Down-sampled }}+M_{\text {Touch }} \times N_{\text {Re gional-scan }} ; \\
& N_{\text {Down-sampled }}=P_{\text {Down-sampled }} \times N_{\text {Sensor }} ;
\end{aligned}
$$

where $N_{\text {Total }}$ denotes the total number of measured sensors and $N_{\text {Down-sampled }}$ is the number of randomly selected sensors. $P_{\text {Down-sampled }}$ and $N_{\text {sensor }}$ are the percentage of sampled sensors and total number of sensors in a touch panel, respectively, and $M_{\text {Touch }}$ is the number of touch events. The relationship between $P_{\text {Down-sampled, }}, N_{\text {Regional-scan }}$ and $N_{\text {Down-sampled }}$ in this work is illustrated in Table II.

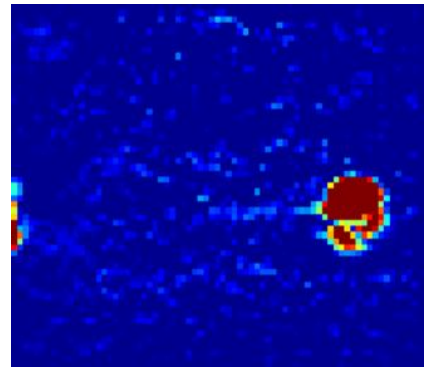

(a)

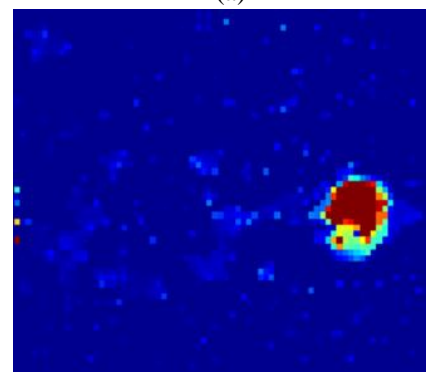

(c)

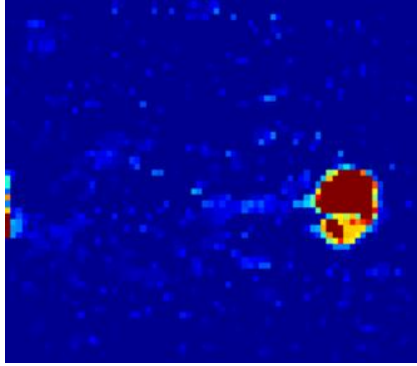

(b)

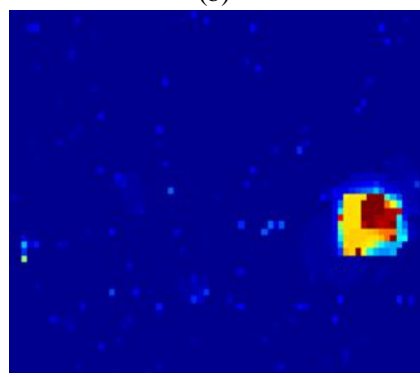

(d)
Fig. 4. (a) Original signal; (b), (c) and (d) are reconstructed signals with $50 \%, 30 \%$ and $10 \%$ of original electrode pads.

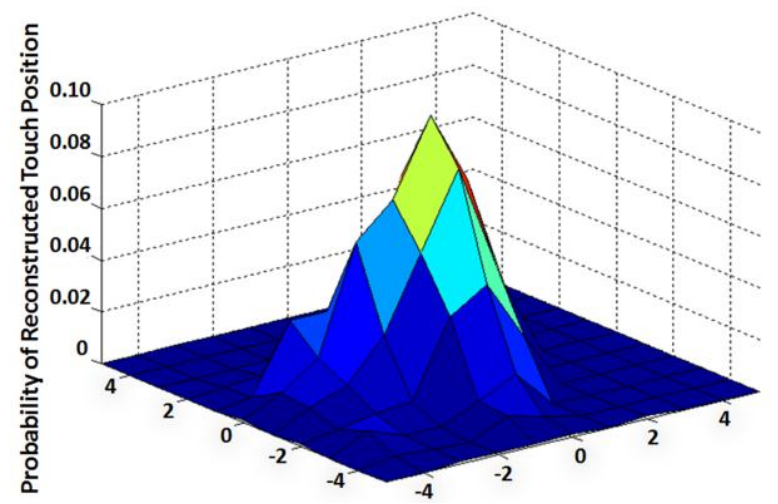

(a)

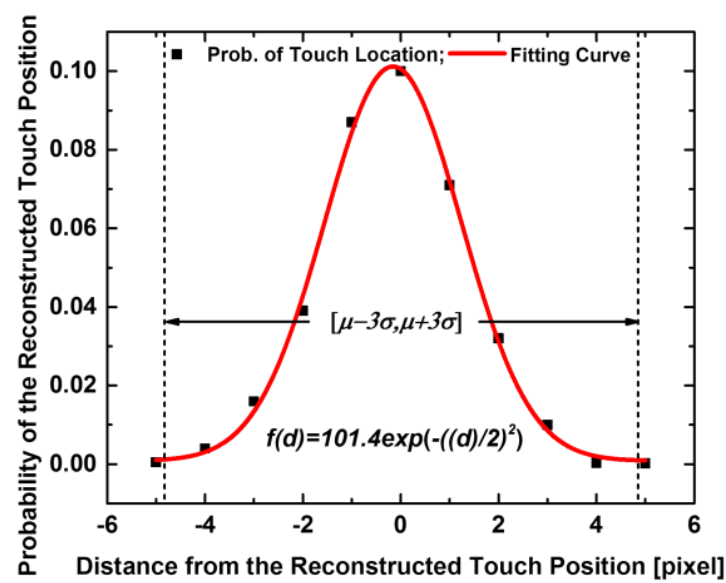

(b)

Fig. 5. (a) Distribution of the reconstructed touch positions; X-Y coordinates indicate the distance between the reconstructed touch position and original touch position. (b) The cross-section with the largest variance of (a). 


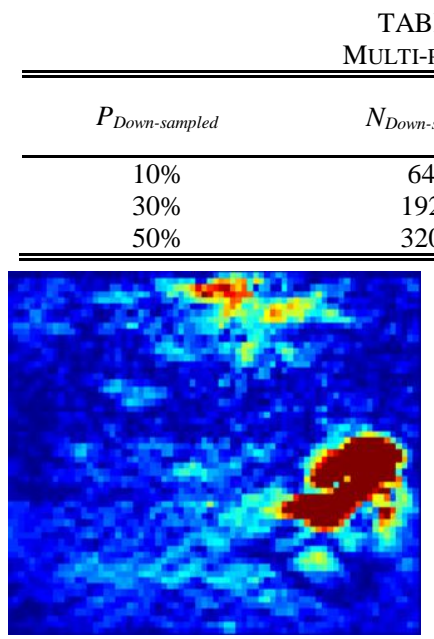

(a)

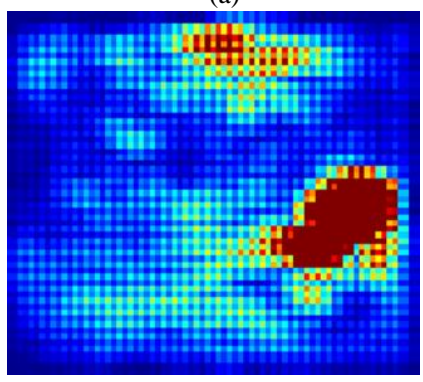

(c)

Fig. 6. (a) original swipe touch based frame, (b) down-sampled touch signal, (c) reconstructed touch signal by using low-pass spatial filtering, (d) regional scanned touch signal.

It is observed that the presented algorithm is more suited to large-scale touch panels. For example, for a small-scale touch panel there are $320(20 \times 16)$ touch sensors and $50 \%$ sensors are randomly selected, if greater than 4 touch events happen $N_{\text {Total }}$ may be bigger than $N_{\text {Sensor }}$ It is possible that a noise spike is sampled and remained in the reconstructed touch signal. However this will not affect touch detection accuracy, as in touch decision function, noise reduction methods are normally applied, thus this will not contribute to additional regional scan and cause further cost.

\section{B. Experimental and Simulation Results for Fixed Pattern Down-sampling}

By analyzing the characteristic of the frame after zero-insertion $\left(f_{\text {zero-insertion }}\right)$, the filter size has to be $(2 n+3) \times$ $(2 n+3)$, where $n$ is a positive odd integer. In this paper, a $5 \times 5$ average filter is employed.

A swipe touch frame is illustrated in Fig. 6 with the corresponding reconstructed frames using a low-pass spatial filter as an example, based on which it can be observed that the touch event's low frequency spatial property is reconstructed. After applying the spatial low-pass filter, an additional benefit is the boost in ratio between the touch signal to the peak noise spike, which in our experiments is $5.4 \mathrm{~dB}$ on average. The regional scan range is determined by the reconstruction quality, which is related with the touch signal properties (shape and position) and the size of spatial LPF. In the above, the determined filter size to smooth the noise spikes is $5 \times 5$, based

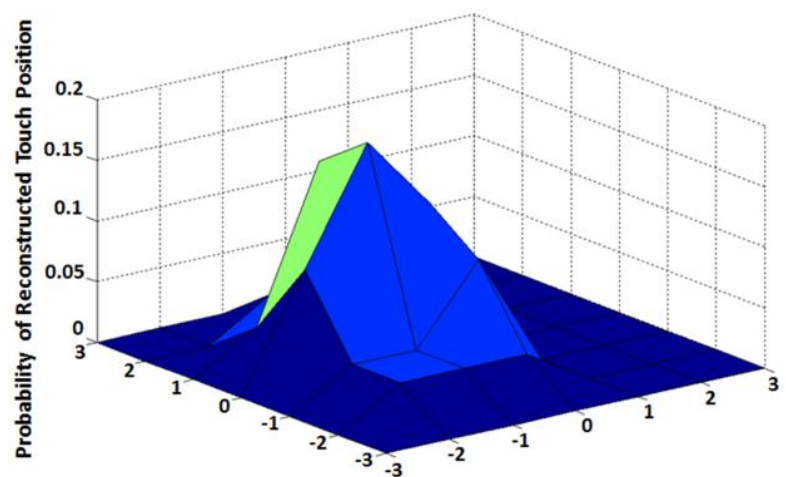

(a)

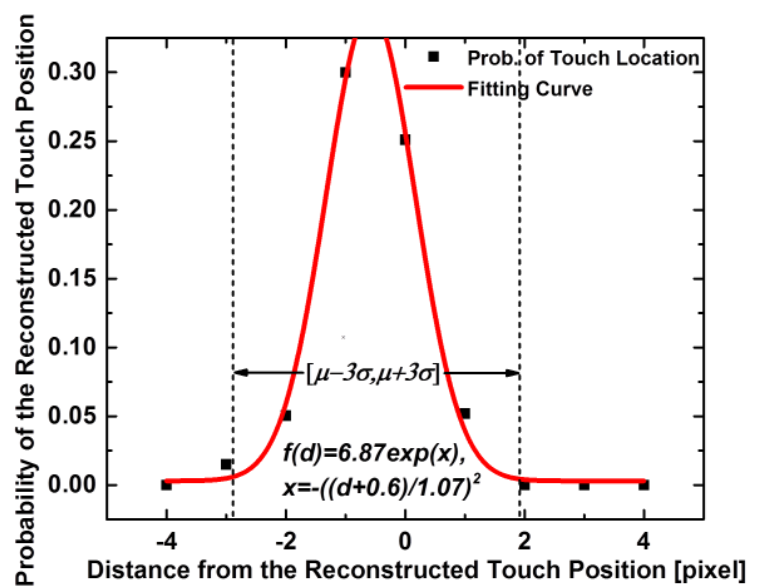

(b)

Fig. 7. (a) Distribution of the reconstructed touch positions; X-Y coordinates indicate the distance between the reconstructed touch position and original touch position. (b) The cross-section with the largest variance of (a).

on which the distribution of the estimated touch positions are shown in Fig. 7. It can be observed that the distribution shape follows a Gaussian (Fig. 7 (a)). Taking the cross-section of the direction with the largest variance (Fig. 7 (b)), we analyze the probability of the changed distance, which can be modeled as

$f(d)=6.87 \exp \left(-((d+0.6) / 1.07)^{2}\right)$

where $d$ is the distance between the reconstructed touch position and the original touch position. Align to the assumption made above, the maximum changed distance is 2 . From Eq. 7, it can be shown that to acquire the accurate single touch position, only 49 electrode pads centered at the peak touch value location are required to be regionally scanned. The number of totally measured sensors is 1649 . In contrast, 6400 sensors need to be read previously. The analysis of the number of measured sensors for multi-touch events is the same as discussed in random pattern down-sampling section.

\section{Algorithm Time and Energy Budget}

If the minimum $\ell_{1}$ norm reconstruction algorithm is employed, the computation complexity is $O\left(\mathrm{Nlog}_{2} N\right)$ [28], 
which indicates that approximately $80 \mathrm{~K}$ multiplications are needed to reconstruct the signal. For a GHz range processor, the calculation time is about $80 \mu \mathrm{s}$. Compared to the duration of a touch event, which is assumed to be $0.1 \mathrm{~s}$, and the scanning interval (17 ms), the calculation time is negligible. Commercial embedded processors achieve a power efficiency of over $20 \mathrm{MIPS} / \mathrm{mW}$ [43], which means that the power consumption is around $25 \mu \mathrm{W}$. The energy required for measuring one sensor is approximately $1 / 30 \mu \mathrm{J}$ [28]. For the experiment test-bed, the power consumption is around $12.8 \mathrm{~mW}$. Therefore, the power consumption of the compressive sensing reconstruction is insignificant. If only $30 \%$ of the electrodes are measured, then around $8.79 \mathrm{~mW}$ can be saved.

If touch information is the most important consideration then iterations of regional scans can be performed to obtain more detailed information. For example, our experiment was carried out on an $80 \times 80$ touch screen panel with a scan rate of $60 \mathrm{~Hz}$, thus around $2.6 \mu \mathrm{s}$ was required to read one sensor. If in the down-sampled stage $50 \%$ of the sensors are measured, roughly $8.4 \mathrm{~ms}$ is needed to use the reconstruction algorithm, after which the rest of the time can be used for a regional scan. One regional scan takes about $0.2 \mathrm{~ms}$, thus during one touch event approximately 40 regional scans can be performed. As more time is used to read the sensors, noise can be averaged. The above relationship can be expressed as:

$$
T_{\text {Frame }} \geq T_{\text {Down-sampled }}+M_{\text {Touch }} N_{\text {Times }} N_{\text {Regiona-scan }} T_{\text {Sensor }} \text {; }
$$

where $T_{\text {Frame }}$ is the time to scan the original frame (e.g. $16.7 \mathrm{~ms}$ in this paper), $T_{\text {Down-sampled }}$ the time to read the down-sampled sensors and $T_{\text {Sensor }}$ the time to measure one sensor.

It should be noted that stylus touches were used in our experiments (multi-pad and rows-and-columns), which cover more sensors than a typical finger touch (e.g. $3 \times 3$ ). The radius of the regional scan area for a finger touch can be limited to 1 pixel. Details of this are currently being investigated.

\section{CONCLUSION}

This paper presents a down-sampling based fast readout technique for multi-pad and rows-and-columns capacitance TSPs so to achieve fast readout and low power consumption. By down-sampling the touch sensors and reconstructing the touch related frame, a possible touch position is estimated. To acquire accurate touch information, a regional scan is then conducted around the estimated touch position. Through the presented technique, the savings in power consumption is $8.79 \mathrm{~mW}(68.7 \%)$ and the readout speed is boosted by $11.3 \mathrm{~ms}$ (68\%) without compromising detection accuracy.

\section{REFERENCES}

[1] L. Zhang, J. Saboune and A. E. Saddik, "Transforming a regular screen into a touch screen using a single webcam", IEEE J. Display Technol., vol.10, no. 8, pp. 647-659, Aug. 2014.

[2] J. Lee, M. T. Cole, J. C. S. Lai and A. Nathan, "An analysis of electrode patterns in capacitive touch screen panels", IEEE J. Display Technol., vol. 10, no.5, pp. 362-366. May 2014.

[3] W. C. Westerman, S. Hotelling, "Perpheral pixel noise reduction", U.S. Patent US7643010 B2, Jan 2010.
[4] S. Hotelling, J. Strickon, B. Huppi, I. Chaudhri, G. Christie, B. Ording, D. Kerr and H. Ive, "Gestures for touch sensitive input devices", U.S. Patent US20060026521 A1, Feb. 2006.

[5] B. R. Land and S. P. Hotelling, "Error compensation for multi-touch surfaces”, U.S. Patent US7692638 B2, Apr. 2010.

[6] Z. Baharav and R. Kakarala, "Capacitive touch sensing: Signal and image algorithms", Proc. SPIE Conf. on Comput. Imaging, Feb. 2011.

[7] H. Akhtar and R. Kakarala, "A methodology for evaluating accuracy of capacitive touch sensing grid patterns", IEEE J. Display Technol., vol.10, no. 8, Aug. 2014.

[8] T. Wang, Y. Ko, C. Lai, "Gesture detecting method capable of filtering panel mistouch", U.S. Patent US20130093692, Apr. 2013.

[9] S. Hagermoser, "Harnessing the power of projected capacitive technology using unfiltered touchscreen data", 3M Touch Systems, 2014.

[10] J. Jordan, "Sensing and defining an input object", U.S. Patent US20110057670 A1, Mar. 2011.

[11] I. Guarneri, A. Capra, G. M. Farinella and S. Battiato, "Shape recognition for capacitive touch display", SPIE Electronic Imaging, Mar. 2013.

[12] W. C. Westerman, "Image jaggedness filter for determining whether to perform baseline calculations", U.S. Patent, US20090174688 A1, Jul. 2009.

[13] W. C. Westerman, "Multi-touch input discrimination", U.S. Patent, US20080158185 A1.

[14] Guarneri, I., Capra, C., Castorina, A., Battiato, S., Farinella, M.,G., "PCA based shape recognition for capacitive touch display", IEEE ICCE, pp. $1-2,2013$.

[15] J. Yang, S. Park, J. Choi, H. Kim, C. B. Park, S. Ryu and G. Cho, "A noise-immune high-speed readout circuit for in-cell touch screen panels", IEEE Trans. Circuits Syst. I, Reg. Papers, vol. 60, no. 7, pp. 1800-1809, Jul. 2013.

[16] S. P. Hotelling, D. T. Amm, M. Lammers, J. Orchard, B. M. King, O. S. Leung, D. Teoman, "Ground detection for touch sensitive device", U.S. Patent WO2011008610 A1, Jan. 2011.

[17] H. S. Lee, T. J. Park and K. J. Kim, "Touch sensing method and touch sensing device", U.S. Patent US20140139478 A1, May 2014.

[18] J. Zhang, Y. Guo and L. Mo, "Multi-touch detection method for capacitive touch screens", U.S. Patent, US20110221701 A1, Sep. 2011.

[19] B. R. Land, M. Yousefpor and S. P. Hotelling, "Negative pixel compensation", U.S. Patent, US8570301 B2, Oct. 2013.

[20] J. Liu, L. Liu, C. Chang and S. Tai, "Touch device and driving method of touch panel thereof", U.S. Patent US20140145965 A1, May 2014.

[21] H. Philipp, "Capacitive sensor and array", U.S. Patent US6452514 B1, Sep. 2002.

[22] S. Gao, J. Lai, C. Micou and A. Nathan, "Reduction of Common-mode Noise and Global Offsets in Touch Screens by Correlated Double Sampling ”, IEEE J. Display Technol. DOI 10.1109/JDT.2016.2515847.

[23] S. Gao, D. McLean, J. Lai, C. Micou and A. Nathan, "Reduction of Noise Spikes in Touch Screen Systems by Low Pass Spatial Filtering", IEEE J. Display Technol. DOI 10.1109/JDT.2016.2550519.

[24] D. L. Donoho, "Compressed sensing", IEEE Trans. Info. Theory, vol. 52, no. 4, July, 2006

[25] Y. Tsaig, D. L. Donoho, "Extensions of compressed sensing", Signal Processing, vol. 86, issue 3, pp. 549-571, Mar. 2006.

[26] M. A. T. Figueiredo, R. D. Nowak and S. J. Wright, "Gradient projection for sparse reconstruction: application to compressed sensing and other inverse problems", IEEE J. Sel. Topics in Signal Process., vol. 1, no. 4, pp. 586-597, Dec. 2007

[27] R. Baraniuk, "Compressive sensing", IEEE Signal Process. Mag., vol. 24, pp. 118-124, July 2007.

[28] C. Luo, J. McClellan, M. A. Borkar and A. Redfem, "Sparse touch sensing for capacitive touch screens", IEEE Proc. ICASSP, pp. 2545-2548, Mar. 2012

[29] C. Luo, M. A. Borkar and A. J. Redfem, "System and method for sparse touch sensing on capacitive touch screen", U.S. Patent, US20130257783 A1, Oct. 2013.

[30] C. Luo, M. A. Bokar, A. J. Redfern, and J. H. McClellan, "Compressive sensing for sparse touch detection on capacitive touch screens", IEEE Journal on Emerging and Selected Topics in Circuit and Systems, vol. 2, no. 3, pp. 639-648, Sept. 2012

[31] M. Yaghoobi, S. McLaughlin and M. E. Davis, "Super-resolution sparse projected capacitive multitouch sensing", IET ISPC, pp. 1-3, 2013.

[32] H. Nyquist, "Certain topics in telegraph transmission theory", Transactions on American Institute of Electrical Engineers, vol. 47, issue 2, pp. 617-644, 1928 
[33] R. Baraniuk, M. Davenport, M. R. DeVore and M. Wakin, "A simple proof of the restricted isometry property for random matrices", Constr. Approx., vol. 28, no. 3, pp. 253-263, Jan. 2008.

[34] M. Rudelson and R. Vershynin, "On sparse reconstruction from Fourier and Gaussian measurements", Comm. Pure and Appl. Math., vol. 61, pp. 1025-1045, Aug. 2008.

[35] W. Rudin, "Function theory in the unit ball of Cn", Springer Science \& Business Media, 2009.

[36] G. Rudolph, "Convergence analysis of canonical genetic algorithms", IEEE Trans. Neural Networks, vol. 5, no. 1, pp. 96-101, Jan, 1994.

[37] E. Candés, J. Romberg and T. Tao, "Robust uncertainty principles: Exact signal reconstruction from highly incomplete frequency information", IEEE Trans. Info. Theory, vol. 52, no. 2, pp. 489-509, Feb. 2006.

[38] D. L. Donoho, "For most large underdetermined systems of linear equations the minimal $\ell 1$-norm solution is also the sparsest solution", Comm. Pure and Appl. Math., vol.59, no.6, pp. 797-829, June 2006.

[39] S. G. Mallat and Z. Zhang, "Matching pursuits with time-frequency dictionaries", IEEE Trans. Signal Process, vol. 41, no. 12 pp. 3397-3415, Dec. 1993.

[40] Y. Saad, "Iterative methods for sparse linear systems," SIAM, 2003.

[41] J. A. Tropp, and C. G. Anna, "Signal recovery from random measurements via orthogonal matching pursuit", IEEE Trans. Info. Theory, vol. 53, no.12, pp. 4655-4666, Dec. 2007.

[42] F. William, "An introduction to probability theory and its applications", John Wiley and Sons, 1964.

[43] ARM, "Cortex-a5 processor performance," [Online], Available: http://www.arm.com/products/processors/cortex-a/cortex-a5.php

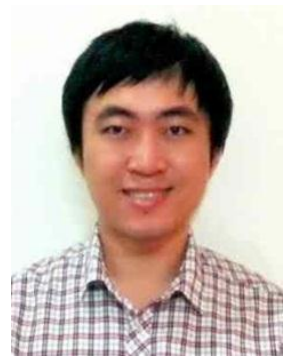

Shuo Gao received the M.Sc. in electrical engineering from the University of Ottawa, Canada, 2013. He is currently a Ph.D. student in the Hetero-Genesys Laboratory, University of Cambridge. Previously he worked as an optical fiber system engineer at Ciena Corporation, Ottawa, Canada. His research interests include touch interactivity and RF system for flexible electronics.

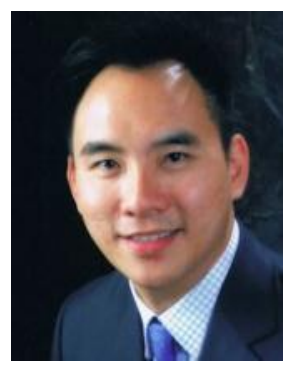

Jackson Lai received the Ph.D. in electrical engineering from the University of Waterloo, Canada, in 2007. He is currently a Senior Display Technology Developer at Blackberry. Previously, he has held positions such as Device and Circuit Engineer in Carestream Health, as well as Lead Display Circuit Designer in Ignis Innovation Inc. His research interests include imaging circuits, large area electronics, as well as system on panel design.

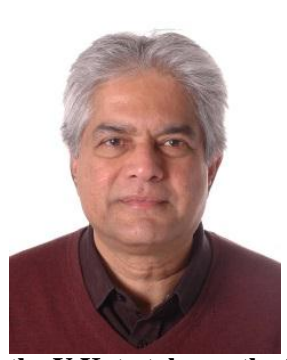

Arokia Nathan (S'84-M'87-SM'99-F'10) received the Ph.D. degree in electrical engineering from the University of Alberta. Following post-doctoral years at LSI Logic Corp., USA, and ETH Zurich, Switzerland, he joined the University of Waterloo, Canada, where he held the DALSA/NSERC Industrial Research Chair in sensor technology and subsequently the Canada Research Chair in nano-scale flexible circuits. He was a recipient of the 2001 NSERC E.W.R. Steacie Fellowship. In 2006, he moved to the U.K. to take up the Sumitomo Chair of Nanotechnology at the London Centre for Nanotechnology, University College London, where he received the Royal Society Wolfson Research Merit Award. He has held Visiting Professor appointments at the Physical Electronics Laboratory, ETH Zürich and the Engineering Department, Cambridge University, U.K. He holds the Chair of Photonic Systems and Displays in the Department of Engineering, Cambridge University. He has published over 500 papers in the field of sensor technology, CAD, thin film transistor electronics, and is a co-author of four books. He has over 50 patents filed/awarded and has founded/co-founded four spin-off companies. He serves on technical committees and editorial boards in various capacities. Dr. Nathan is a Chartered Engineer (U.K.), Fellow of the Institution of Engineering and Technology (UK), Fellow of IEEE (USA), and an IEEE/EDS Distinguished Lecture. 